\title{
Voltage Fluctuation to Current Converter with Coulomb-Coupled Quantum Dots
}

\author{
F. Hartmann, ${ }^{1, *}$ P. Pfeffer, ${ }^{1}$ S. Höfling,,${ }^{1,2}$ M. Kamp, ${ }^{1}$ and L. Worschech ${ }^{1}$ \\ ${ }^{1}$ Technische Physik, Universität Würzburg, Physikalisches Institut and Wilhelm Conrad Röntgen Research Center for Complex \\ Material Systems, Am Hubland, D-97074 Würzburg, Germany \\ ${ }^{2}$ SUPA, School of Physics and Astronomy, University of St. Andrews, St. Andrews, KY16 9SS, United Kingdom
}

(Received 10 December 2014; published 10 April 2015)

\begin{abstract}
We study the rectification of voltage fluctuations in a system consisting of two Coulomb-coupled quantum dots. The first quantum dot is connected to a reservoir where voltage fluctuations are supplied and the second one is attached to two separate leads via asymmetric and energy-dependent transport barriers. We observe a rectified output current through the second quantum dot depending quadratically on the noise amplitude supplied to the other Coulomb-coupled quantum dot. The current magnitude and direction can be switched by external gates, and maximum output currents are found in the nA region. The rectification delivers output powers in the $\mathrm{pW}$ region. Future devices derived from our sample may be applied for energy harvesting on the nanoscale beneficial for autonomous and energy-efficient electronic applications.
\end{abstract}

Extracting work from random fluctuations by energy conversion to a unidirectional particle flow is a key enabling technology and has consequently triggered substantial experimental and theoretical work [1-4]. The exploitation of temperature and fluctuation gradients for energy harvesting has led to new concepts such as Brownian and Büttiker-Landauer motors [5-8], phonon rectifiers $[9,10]$, and piezoelectric nanogenerators [11-13]. Challenging factors in miniaturizing heat engines are an efficient energy conversion and the maintenance of welldefined hot and cold spots [14]. Quantum dot structures are among the smallest possible heat engines conceived thus far. Pioneering work in this field was conducted by, among others, Molenkamp et al., who measured the Seebeck voltage of single quantum dots (QDs) and quantum point contacts (QPCs) [15-17]. In recent years, research concerning heat engines based on QDs and QPCs followed [18-22]. Furthermore, Coulomb-coupled systems attracted attention due to their ability to generate currents in unbiased wires via the Coulomb drag [23-25]. A striking proposal combining rectifying effects with QDs was recently made by Sánchez et al., who showed that two capacitively coupled QDs connected to electron reservoirs operated in the Coulomb-blockade regime can act as a rectifier that transfers each energy quantum that passes from one to the other QD to the motion of single electrons (i.e., to charge quanta) [26]. Notably, the heat and charge current directions are decoupled in the proposed system. Later, Sothmann et al. investigated a similar design based on open QD systems (with conductances higher than the conductance quantum) exhibiting higher output currents, which makes this proposal more accessible to experimental realization $[27,28]$. Furthermore, it combines maximum output power as well as maximum efficiency at the same electrostatic configuration, which is in contrast to
Coulomb-blockade systems, where maximum efficiency theoretically occurs at zero output power [26].

In this Letter, we present a system that converts voltage fluctuations into a directed current depending on the fluctuation's amplitude and whose direction and magnitude can be manipulated via external gates.

The operation principle of the device is illustrated in Fig. 1(a) where the upper $\mathrm{QD}_{t}$ is represented as the cavity

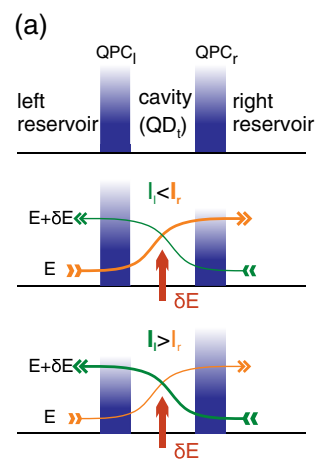

FIG. 1 (color online). (b)

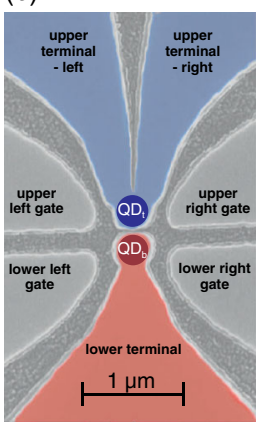

(c)

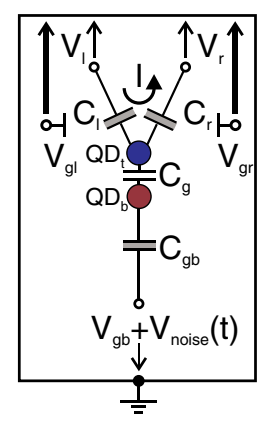
Designation of system components. Center: Asymmetric configuration with charge current flow to the right. Bottom: Asymmetric configuration with charge current flow to the left. For details see main text. (b) Electron microscopy image of the sample. The top, current carrying part of the system is shaded in blue, the bottom part, where the voltage fluctuations are supplied, in red. The respective $\mathrm{QDs}_{\mathrm{QD}}$ and $\mathrm{QD}_{b}$ are highlighted in dark blue and dark red. (c) Equivalent circuit with corresponding capacitances. The current through the upper part is measured via a picoamperemeter. The two upper side gates and their voltages $V_{\mathrm{gl}}$ and $V_{\mathrm{gr}}$ control the conductances of the left and right channel, whereas $V_{\mathrm{gb}}$ influences both channels almost equally and shifts the QD's energy levels. $V_{\text {noise }}$ can be added to $V_{\mathrm{gb}}$ and provides the fluctuations which the device is able to rectify. 
connected via QPCs to electron reservoirs. The lower $\mathrm{QD}_{b}$ serves as the energy source, which is mediated via Coulomb interaction to electrons in $\mathrm{QD}_{t}$, thus raising their energy by $\delta E$. The two blue columns in the sketch represent the transmissivities of the two QPCs. The brighter the part of a blue column, the higher the transmissivity at the respective energy. Horizontal arrows illustrate the current flow through $\mathrm{QD}_{t}$ with bigger arrows symbolizing higher currents. Vertical arrows stand for the energy supply $\delta E$ to the upper cavity $\mathrm{QD}_{t}$. Provided that the whole electron path [thicker orange arrow in the middle panel of Fig. 1(a)] for electrons entering the cavity from the left with a lower energy, then increasing their energy by $\delta E$ in the cavity, and finally leaving the cavity through the right QPC has a larger total transmissivity compared to electron path with the opposite direction (thin green arrow), $\mathrm{QD}_{t}$ operates as the rectifier. By changing the transmissivities by external gates or a proper asymmetric design of the QPCs, the rectified current direction can be inverted (lower panel). In this device concept, no current flows between the energy supplying $\mathrm{QD}_{b}$ and the rectifier $\mathrm{QD}_{t}$.

For the realization of such structures, we grew a modulation doped GaAs/AlGaAs heterostructure with a high mobility two-dimensional electron gas (2DEG) located about $80 \mathrm{~nm}$ below the surface by molecular beam epitaxy. Electron beam lithography and dry chemical etching techniques were used to etch trenches that cut through the 2DEG, defining the device and also outer side gates. Figure 1(b) shows an electron microscopy image of such a sample. For clarity, $\mathrm{QD}_{t}$ connected to the left and right upper terminals is dyed in blue. $\mathrm{QD}_{b}$ connected to the lower terminal is dyed in red. The upper left and right gates were used to control the transmittivities of the $\mathrm{QD}_{t}$ 's contacts with the applied gate voltages $V_{\mathrm{gl}}$ and $V_{\mathrm{gr}}$. The lower gates were directly connected to ground. $\mathrm{QD}_{t}$ is connected to the left and right voltage sources with voltages $V_{l}$ and $V_{r}$, respectively. $\mathrm{QD}_{b}$ is connected to the lower reservoir where controllable voltage fluctuations due to a noise source occur. Figure 1(c) shows the equivalent circuit.

Similar to a Smoluchowski-Feynman ratchet [14], the analyzed converter can only work when the magnitude of fluctuations differs across the device, meaning in our case, when the fluctuations in $\mathrm{QD}_{b}$ exceed those in the left and right reservoir. In order to prevent particle exchange between the two subsystems, we separated them using etched trenches of about $150 \mathrm{~nm}$. Thus, electronic fluctuations in $\mathrm{QD}_{b}$ are mostly conveyed to the upper subsystem by means of Coulomb coupling to $\mathrm{QD}_{t}$.

Figure 1(c) depicts the circuit diagram of the device. The QDs are coupled to their respective reservoirs by leads, whereas the QDs are coupled to each other by means of the capacitance $C_{g}$. The upper reservoirs can be biased by $V_{l r}=V_{l}-V_{r}$, and the current through the contacts was measured using a low noise picoamperemeter. The bottom $\mathrm{QD}$ can serve as local gate of $\mathrm{QD}_{t}$ by tuning the voltage
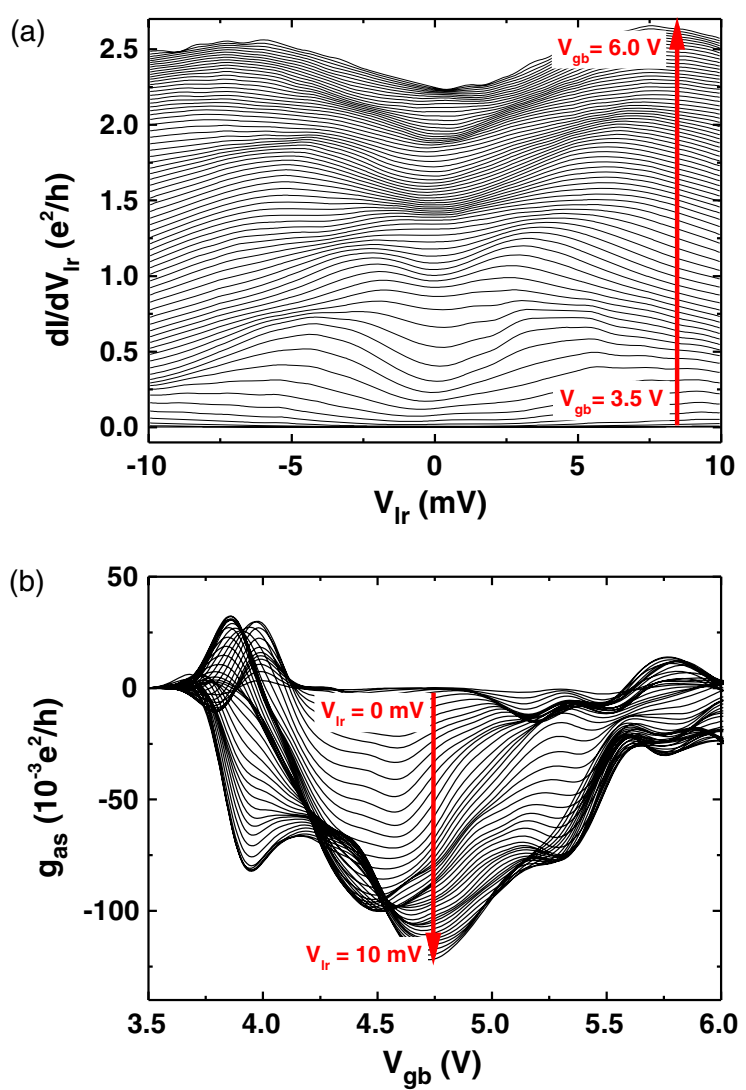

FIG. 2 (color online). (a) Differential conductance traces for $V_{\mathrm{gb}}$ from 3.5 to $6 \mathrm{~V}$ against $V_{l r}$. Dark regions where the traces lie close to each other signify stable conductances. (b) Conductance asymmetry $g_{\mathrm{as}}$ against $V_{\mathrm{gb}}$ for $V_{l r}$ ranging from 0 to $10 \mathrm{mV}$.

$V_{\mathrm{gb}}$. All experiments reported here were conducted at $4.2 \mathrm{~K}$ in the dark by immersing the sample in liquid helium.

Figure 2(a) shows several conductance traces $d I / d V_{l r}$, with $I$ being the current through $\mathrm{QD}_{t}$ for different $V_{\mathrm{gb}}$ ranging from 3.5 to $6 \mathrm{~V}$ while $V_{g l}=V_{g r}=0 \mathrm{~V}$. One can see several darker regions of a high trace density where the conductance is relatively stable against gate voltage changes. For small bias voltages the conductance at $V_{\mathrm{gb}}=$ 4.7 $\mathrm{V}$ lies inside a stable region reaching a value of about $1.5 e^{2} / h$.

For the purpose of observing the upper system part's ability to rectify fluctuating currents, it is useful to define the conductance asymmetry

$$
g_{\mathrm{as}}\left(V_{l r}\right)=1 / 2\left[d I / d V_{l r}\left(-V_{l r}\right)-d I / d V_{l r}\left(+V_{l r}\right)\right],
$$

which is displayed in Fig. 2(b) versus $V_{\mathrm{gb}}$ (different traces). Varying $V_{\mathrm{gb}}$ from small to higher values shifts the system from a configuration with small and sign-changing $g_{\text {as }}$ values to a region exhibiting large negative $g_{\text {as }}$ when $V_{\mathrm{gb}} \approx 4.7 \mathrm{~V}$. For even larger $V_{\mathrm{gb}}$, small and sign-changing $g_{\text {as }}$ can be seen again. Large positive or negative values of $g_{\text {as }}$ reflect transmission asymmetries and can be found in 
the region between $V_{\mathrm{gb}}=3.8$ and $5.5 \mathrm{~V}$. Thus, the operating point was set to this open QD regime at $V_{\mathrm{gb}}=$ $4.7 \mathrm{~V}$ in the following experiment.

For the controlled generation of voltage fluctuations in the lower reservoir, a Gaussian-distributed and spectrally flat noise source with a root mean square noise amplitude $\sigma_{\text {noise }}$ and a cutoff frequency $f=20 \mathrm{MHz}$ was used. The noise was added to the static gate voltage $V_{\mathrm{gb}}$ resulting in an overall voltage at the lower reservoir of $V_{\mathrm{ov}}(t)=V_{\mathrm{gb}}+V_{\text {noise }}(t)$. In order to keep the central electrostatic potential of $\mathrm{QD}_{t}$ constant while asymmetrically controlling the conductances of the left and right channel $G_{l}$ and $G_{r}$ (reducing $G_{l}$ increases $G_{r}$ and vice versa), $V_{\mathrm{gl}}$ and $V_{\mathrm{gr}}$ were varied in push-pull configuration, i.e., $\Delta V_{\mathrm{gl}}=-\Delta V_{\mathrm{gr}}$, with $V_{\mathrm{gl}}+V_{\mathrm{gr}}=0$ in our case. In Fig. 3(a) the rectified current through $\mathrm{QD}_{t}$ is plotted for different noise amplitudes ranging from $\sigma_{\text {noise }}=7.6$ to $144 \mathrm{mV}$ for $V_{l r}=0 \mathrm{~V}$. At zero $\sigma_{\text {noise }}$, no rectified current $I$ could be measured, but applying noise and sweeping the side gate voltages allows detection and control of the current flow. Starting with a gate voltage configuration of $V_{\mathrm{gl}}=-V_{\mathrm{gr}}=-2 \mathrm{~V}$ and increasing $\Delta V_{\mathrm{gl}}=-\Delta V_{\mathrm{gr}}$ initially increases the current from zero to a maximal value
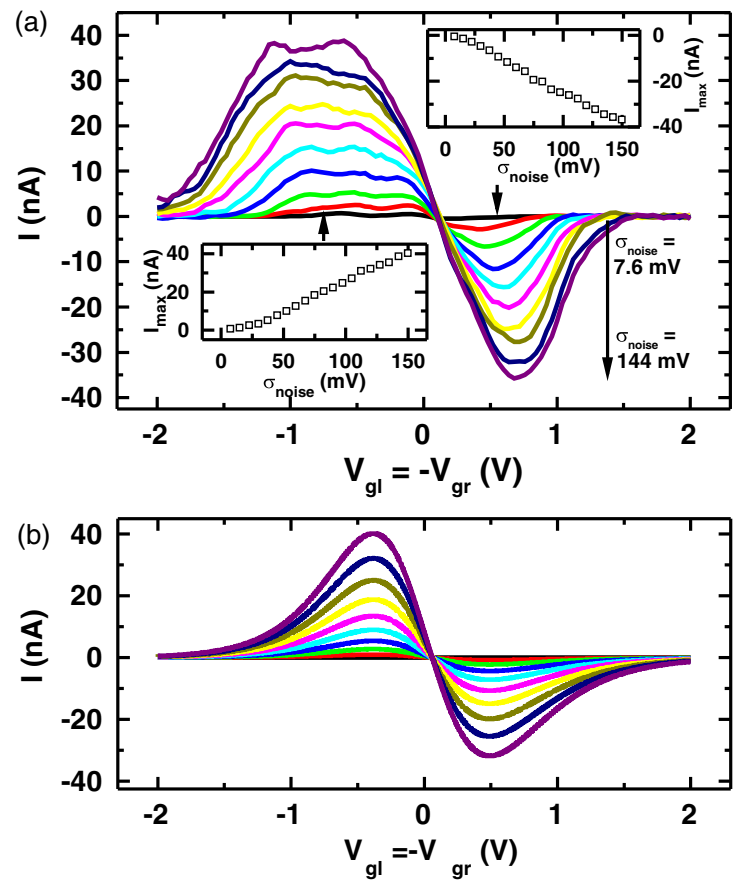

FIG. 3 (color online). (a) Experimental results of the noise rectification. The output current is plotted against $V_{\mathrm{gl}}=-V_{\mathrm{gr}}$ (push-pull) for noise amplitudes varied from 7.6 to $144 \mathrm{mV}$ in steps of $15 \mathrm{mV}$. $V_{l r}=0 \mathrm{~V}, V_{\mathrm{gb}}=4.7 \mathrm{~V}$. $\sigma_{\text {noise }}$ controls the maximum current amplitude, while the side gates are able to influence the current flow's direction. The insets show the current maxima $I_{\max }$ in dependence on $\sigma_{\text {noise }}$ up to $150 \mathrm{mV}$. (b) Simulated current rectification. $I$ versus $V_{\mathrm{gl}}=-V_{\mathrm{gr}}$ (pushpull) for different noise amplitudes. that depends on the applied noise. Thereafter, $I$ decreases again and vanishes completely at around $V_{\mathrm{gl}}=0.01 \mathrm{~V}$ (independently of $\sigma_{\text {noise }}$ ). At $V_{\mathrm{gl}}=V_{\mathrm{gr}}=0$, currents of up to $10 \mathrm{nA}$ can be measured, which indicates that also via a proper asymmetric design of $\mathrm{QD}_{t}$ contacts a rectified current can be generated and thus side gates for application of an in-plane electric field are, in principle, not necessary to achieve rectification. A further increase of $V_{\mathrm{gl}}$ changes the current direction. $I$, therefore, becomes negative, reaches a minimum, and finally increases back to zero again. The disappearance of $I$ below and above certain side gate configurations can be understood bearing in mind that the push-pull method closes one channel while opening the other one [29]. The insets of Fig. 3(a) present the maximum currents for both directions in dependence on the noise amplitude $\sigma_{\text {noise }}$.

A simulated model of the rectification currents after Eqs. (4) and (5) in Ref. [27] can be seen in Fig. 3(b). The theoretical curves shown there have been modeled using the following relations and demonstrate the inversion of the critical rectification parameter $\Lambda$ by changing the gate voltages $V_{i}(i=g r, g l$, left and right side gate $)$ in push-pull fashion. According to Ref. [27], the rectified current I through the upper quantum dot system can be expressed as

$$
I=\frac{\Lambda}{\tau_{R C}} k_{B} \Delta T
$$

Here, $\Delta T$ denotes the temperature difference between the upper quantum dot system at temperature $T_{C}$ (cold) and the bottom quantum dot system at temperature $T_{H}$ (hot), $k_{B}$ is the Boltzmann constant, $\tau_{R C}$ is the effective $R C$ time of the (double) cavity with the effective capacitance $C_{\text {eff }}$ and conductance $G_{\text {eff }}$ (cf. Ref. [27]). We assume that the energy difference $k_{B} \Delta T$ between the two systems equals the energy stored on the capacitor $E_{\text {cap }}$, with $E_{\text {cap }}=$ $1 / 2 C_{g} \sigma_{\text {noise }}^{2}\left(C_{g}\right.$ being the capacitance between the two QDs). Thus,

$$
I=1 / 2 \Lambda C_{g} / C_{\text {eff }} G_{\text {eff }} \sigma_{\text {noise }}^{2}
$$

The rectification parameter $\Lambda$ can be written in terms of energy-independent parts $G_{i}=\left(e^{2} / h\right) T_{i}$, with transmission $T_{i}$, and energy-dependent parts $G_{i}^{\prime}=\left(e^{3} / h\right) T_{i}^{\prime}$, with transmission $T_{i}^{\prime}$. Following Ref. [30], the energy-dependent conductance of a QPC is

$$
G\left(E_{n}, T\right)=\frac{e^{2}}{h} \sum_{n=1}^{\infty}\left[1+\exp \left(\frac{E_{n}-E_{F}}{k_{B} T}\right)\right]^{-1},
$$

where $E_{F}$ is the Fermi energy, $E_{n}$ the energy of the bottom of the $n$th subband, and $T$ the temperature. We model the conductance of each channel via Eq. (4), where $E_{F}-E_{n}=e \eta_{i} V_{i}, i=g l$ or $i=g r$, and $\eta_{i}$ corresponds 
to the side gate efficiency for the left (right) gate. Thus, $\eta_{i}$ accounts for the opening or closing of the channel via the gate voltage $V_{i}$. Following Ref. [27], the energy-dependent part of the conductance is modeled with $G_{i}^{\prime}=\left(\partial / \partial V_{i}\right) G_{i}$. Inserting equations for $G_{i}$ and $G_{i}^{\prime}$ for the rectification parameter and using the experimentally determined system parameters results in current-gate voltage traces as depicted in Fig. 3(b). These parameters are $T=4.2 \mathrm{~K}, \eta_{l}=0.12 \%$, $\eta_{r}=0.10 \%, G_{\text {eff }}=e^{2} / h$, and $C_{g} / C_{\text {eff }}=0.32$.

To harvest useful work from the device, it has to power a load or, equivalently, the generated current has to flow against a finite voltage difference. So, to measure the output power $P$, a voltage difference $V_{l r}$ counteracting the current was applied between the channels. $P$ is then given by $P=I V_{l r}$. Figures 4(a) and 4(c) show the measured powers $P$ for both current directions plotted versus $V_{l r}$. The output power has a parabolic dependency on $V_{l r}$ and vanishes at two particular points, one of them being at $V_{l r}=0 \mathrm{~V}$ and the other one occurring at the stopping voltage $V_{\text {st }}$, which depends on $\sigma_{\text {noise }}$. At $V_{l r}=V_{\text {st }}$, the generated current is compensated and $I$ vanishes. The curves reach their maxima at half their corresponding stopping voltages.

In Figs. 4(a) and 4(c), $\sigma_{\text {noise }}$ was varied from 7.6 to $150 \mathrm{mV}$. Increasing $\sigma_{\text {noise }}$ results in an increase of both $P_{\max }$ and $V_{\text {st }}$. For $\sigma_{\text {noise }}=150 \mathrm{mV}$, the latter shifts up to $-2.3 \mathrm{mV}$ in the case of positive currents and up to $2.1 \mathrm{mV}$ in the case of negative currents. Maximum output powers versus $\sigma_{\text {noise }}$ are presented in Fig. 4(b) for positive and in
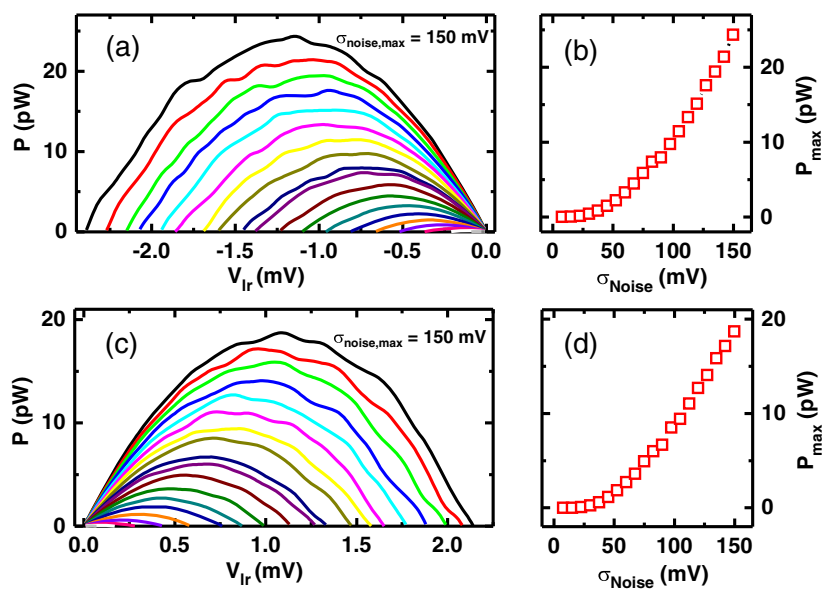

FIG. 4 (color online). (a) Output powers $P$ versus $V_{l r}$ for different $\sigma_{\text {noise }}$ increasing from 7.6 to $150 \mathrm{mV}$ in steps of $15 \mathrm{mV}$. $V_{\mathrm{gl}}=-V_{\mathrm{gr}}=-0.75 \mathrm{~V}$ [cf. Fig. 3(a)]. The power curves feature a parabolic shape, are zero at $V_{l r}=0 \mathrm{~V}$ and at $V_{l r}=V_{\text {st }}$, and reach their maxima at $V_{l r}=V_{\text {st }} / 2$. (b) Maximum output powers $P_{\max }$ against $\sigma_{\text {noise }}$ at $V_{\mathrm{gl}}=-V_{\mathrm{gr}}=-0.75 \mathrm{~V}$. $P_{\max }$ exhibits a quadratic dependence on $\sigma_{\text {noise }}$. (c) Output powers $P$ versus $V_{l r}$ for different $\sigma_{\text {noise }}$ increasing from 7.6 to $150 \mathrm{mV}$ in steps of $15 \mathrm{mV} . V_{\mathrm{gl}}=-V_{\mathrm{gr}}=0.55 \mathrm{~V}$. (d) Maximum output powers $P_{\max }$ against $\sigma_{\text {noise }}$ at $V_{\mathrm{gl}}=-V_{\mathrm{gr}}=0.55 \mathrm{~V} . \quad V_{\mathrm{gb}}=4.7 \mathrm{~V}$ for all figures. The side gate voltage configurations used here are displayed in Fig. 3 via two small black arrows.
Fig. 4(d) for negative currents. Both curves show a quadratic dependency on the noise amplitude and reach maximal values of $P_{\max }=24 \mathrm{pW}$ and $P_{\max }=19 \mathrm{pW}$, respectively.

In summary, we demonstrated a voltage fluctuation to current converter. The output current and power depend on the fluctuation's amplitude and reach maximum values in the $\mathrm{nA}$ and $\mathrm{pW}$ region, respectively. The demonstrated device is a major step to realizing efficient and sustainable electronics by means of energy harvesting in a well-known and widely applicable material system.

The authors gratefully acknowledge financial support from the European Union (FPVII, 2007-2013) under Grant Agreement No. 256959 NANOPOWER and Grant Agreement No. 318287 LANDAUER as well as from the state of Bavaria. Technical assistance by $\mathrm{M}$. Emmerling is gratefully acknowledged. F. Hartmann and P. Pfeffer contributed equally to this work.

* Corresponding author.

fabian.hartmann@physik.uni-wuerzburg.de

[1] H. Linke, T. E. Humphrey, A. Löfgren, A. O. Sushkov, R. Newbury, R. P. Taylor, and P. Omling, Science 286, 2314 (1999).

[2] T. E. Humphrey and H. Linke, Phys. Rev. Lett. 94, 096601 (2005).

[3] R. D. Astumian, Phys. Chem. Chem. Phys. 9, 5067 (2007).

[4] P. Hänggi and F. Marchesoni, Rev. Mod. Phys. 81, 387 (2009).

[5] R. D. Astumian and P. Hanggi, Phys. Today 55, No. 11, 33 (2002).

[6] M. Büttiker, Z. Phys. B 68, 161 (1987).

[7] R. Landauer, J. Stat. Phys. 53, 233 (1988).

[8] A. Gnoli, A. Petri, F. Dalton, G. Pontuale, G. Gradenigo, A. Sarracino, and A. Puglisi, Phys. Rev. Lett. 110, 120601 (2013).

[9] C. W. Chang, D. Okawa, A. Majumdar, and A. Zettl, Science 314, 1121 (2006).

[10] N. Zeng and J.-S. Wang, Phys. Rev. B 78, 024305 (2008).

[11] Z. L. Wang and J. Song, Science 312, 242 (2006).

[12] Y. Qin, X. Wang, and Z. L. Wang, Nature (London) 451, 809 (2008).

[13] S. Xu, B. J. Hansen, and Z. L. Wang, Nat. Commun. 1, 93 (2010).

[14] R. Feynman, R. Leighton, and M. Sands, The Feynman Lectures on Physics: Mainly Electromagnetism and Matter (Addison-Wesley, Boston, 1965).

[15] L. W. Molenkamp, T. Gravier, H. van Houten, O. J. A. Buijk, M. A. A. Mabesoone, and C. T. Foxon, Phys. Rev. Lett. 68, 3765 (1992).

[16] S. F. Godijn, S. Möller, H. Buhmann, L. W. Molenkamp, and S. A. van Langen, Phys. Rev. Lett. 82, 2927 (1999).

[17] R. Scheibner, M. Knig, D. Reuter, A. D. Wieck, C. Gould, H. Buhmann, and L. W. Molenkamp, New J. Phys. 10, 083016 (2008).

[18] N. Nakpathomkun, H. Q. Xu, and H. Linke, Phys. Rev. B 82, 235428 (2010). 
[19] T. Ruokola and T. Ojanen, Phys. Rev. B 83, 241404 (2011).

[20] S. Juergens, F. Haupt, M. Moskalets, and J. Splettstoesser, Phys. Rev. B 87, 245423 (2013).

[21] D. Venturelli, R. Fazio, and V. Giovannetti, Phys. Rev. Lett. 110, 256801 (2013).

[22] F. Haupt, M. Leijnse, H. L. Calvo, L. Classen, J. Splettstoesser, and M. R. Wegewijs, Phys. Status Solidi B 250, 2315 (2013).

[23] M. Yamamoto, M. Stopa, Y. Tokura, Y. Hirayama, and S. Tarucha, Science 313, 204 (2006).

[24] R. Sánchez, R. López, D. Sánchez, and M. Büttiker, Phys. Rev. Lett. 104, 076801 (2010).
[25] D. Laroche, G. Gervais, M. Lilly, and J. Reno, Nat. Nanotechnol. 6, 793 (2011).

[26] R. Sánchez and M. Büttiker, Phys. Rev. B 83, 085428 (2011).

[27] B. Sothmann, R. Sánchez, A. N. Jordan, and M. Büttiker, Phys. Rev. B 85, 205301 (2012).

[28] B. Sothmann, R. Sánchez, and A. N. Jordan, Nanotechnology 26, 032001 (2015).

[29] S. Reitzenstein, L. Worschech, P. Hartmann, M. Kamp, and A. Forchel, Phys. Rev. Lett. 89, 226804 (2002).

[30] C. Beenakker and H. van Houten, in Semiconductor Heterostructures and Nanostructures, Solid State Physics Vol. 44, edited by H. Ehrenreich and D. Turnbull (Academic Press, New York, 1991) pp. 1-228. 